\title{
Time-course pattern of blood 25-hydroxycholecalciferol is a significant predictor of survival outcome in metastatic colorectal cancer: a clinical practice-based study
}

\author{
R. OBERMANNOVA ${ }^{1,2}$, L. DUSEK ${ }^{3}$, K. GREPLOVA ${ }^{2,4}$, J. JARKOVSKY ${ }^{3}$, J. STERBA ${ }^{2,5}$, R. VYZULA ${ }^{1,2}$, R. DEMLOVA ${ }^{2,6}$, L. ZDRAZILOVA-DUBSKA ${ }^{2,4,6}$, \\ D. VALIK $2,4,6, *$
}

\begin{abstract}
${ }^{1}$ Department of Comprehensive Cancer Care, Masaryk Memorial Cancer Institute and Faculty of Medicine, Masaryk University, Brno, Czech Republic; ${ }^{2}$ Regional Centre of Applied Molecular Oncology, Masaryk Memorial Cancer Institute, Brno, Czech Republic; ${ }^{3} I$ Institute of Biostatistics and Analyses, Faculty of Medicine, Masaryk University, Brno, Czech Republic; ${ }^{4}$ Department of Laboratory Medicine, Masaryk Memorial Cancer Institute, Brno, Czech Republic; ${ }^{5}$ Department of Pediatric Oncology, University Hospital Brno and Faculty of Medicine, Masaryk University, Brno, Czech Republic; ${ }^{6}$ Department of Pharmacology, Faculty of Medicine, Masaryk University, Brno, Czech Republic
\end{abstract}

${ }^{*}$ Correspondence: valik@mou.cz

Received March 25, 2015 / Accepted June 15, 2015

\begin{abstract}
Vitamin D deficiency has been implicated in the epidemiology of common malignancies including colorectal cancer. We studied consecutive blood levels of 25-hydroxycholecalciferol (25-OHD) in relation to other clinical and laboratory variables in metastatic colorectal cancer patients to ascertain whether their variations may be prognostic or predictive parameters of survival outcomes. Eighty four patients treated with first-line oxaliplatin-based chemotherapy with or without bevacizumab were included. The patients were enrolled on the intent-to-treat basis considering their performance status, comorbidities and laboratory parameters to be medically apt for intensive chemotherapy. Overall survival and progression-free survival were selected as the primary outcomes. Progression free survival and overall survival medians were 15.4 months and 41.2 months, respectively. The cut-off levels of $40 \mathrm{nmol} / \mathrm{l}$ for $25-\mathrm{OHD}$ and $11 \mu \mathrm{g} / \mathrm{l}$ for first CEA were identified to be clinical decision levels stratifying patients to the respective prognostic groups. We found that the most consistent outcome predictors were i) any patient surgery, ii) CEA and, independently, iii) time-related blood levels of 25OHD. We confirmed fundamental and consistent vitamin D deficiency in metastatic colorectal cancer. We demonstrated that all patients with at least one blood level above $40 \mathrm{nmol} / \mathrm{l}$ versus all below this cut-off showed profound differences in their disease outcomes. The primary disease stage or time to metastatic stage did not influence the predictive power of blood 25-OHD levels, implying that the time-course pattern of 25-OHD but not the first single measurement may be an independent prognostic factor.
\end{abstract}

Key words: colorectal cancer, vitamin D, outcome predictors

Vitamin D has been implicated in the epidemiology of common malignancies including colorectal cancer [1-5]. Several papers reported substantial deficiency of vitamin D in breast and colorectal cancer patients and therapeutic potential of vitamin $\mathrm{D}$ supplementation in cancer has been postulated [6-9] reflecting its pleiotropic biological properties [10, 11]. Blood levels of vitamin $\mathrm{D}$ display vast variations depending on the geographical locality, food habits and way of life $[12,13]$. Reports on colorectal cancer published to date have usually investigated single-sampled levels of 25-hydroxyvitamin D at diagnosis. These studies were mostly inconclusive in demon- strating associations with disease outcomes, presumably due to wide variability of 25-hydroxyvitamin D blood levels [1, 14].

We studied blood 25-hydroxyvitamin D levels and other clinical and laboratory variables in metastatic colorectal cancer patients treated at our institution hypothesizing that consecutive 25-OHD blood level patterns may be more informative than single sampling-based observation as they may better neutralize variations due to common and objective reasons such as seasonal variations. Our specific aims were to find out whether blood 25-OHD levels demonstrate deficiency of vitamin $\mathrm{D}$ and how consistent such deficiency may be in our 
patient population and second, whether blood 25-OHD level time-course patterns are associated with outcome measure in metastatic colorectal cancer.

\section{Patients and methods}

The study was designed as prospective and non-interventional. Our aim was to obtain data from cohort that was homogenous both from the clinical and laboratory viewpoint (Table 1). Inclusion criteria were as follows: i/ metastatic colorectal cancer treated with first-line oxaliplatin-based chemotherapy with or without bevacizumab, and ii/ eligible for intent-to-treat for approach considering their performance status, comorbidities and laboratory parameters so as to be medically apt for intensive chemotherapy with an anti-VEGF component. Exclusion criteria were: i/ PS 3 or ii/ clinical condition not compatible with intensive chemobiotherapy. We enrolled consecutively 84 patients from May 2010 to November 2013. The cohort was not extracted from any other patient set grouped for other purposes such as another clinical trial. Median follow up was 24.2 months. All clinical and laboratory data were obtained from the hospital

Table 1. Basic description of patient cohort according to primary metastatic cancer

\begin{tabular}{|c|c|c|c|c|c|}
\hline \multirow{2}{*}{ Characteristics } & & \multirow[b]{2}{*}{ Total $(\mathrm{N}=84)$} & \multicolumn{2}{|c|}{ Primary metastatic disease } & \multirow{2}{*}{$\mathbf{p}^{1}$} \\
\hline & & & No $(\mathrm{N}=29)$ & Yes $(\mathrm{N}=55)$ & \\
\hline \multirow[t]{4}{*}{ Season - diagnosis } & winter & $\mathrm{N}=22(26.2 \%)$ & $\mathrm{N}=10(34.5 \%)$ & $\mathrm{N}=12(21.8 \%)$ & \multirow{4}{*}{0.604} \\
\hline & spring & $\mathrm{N}=23(27.4 \%)$ & $\mathrm{N}=8(27.6 \%)$ & $\mathrm{N}=15(27.3 \%)$ & \\
\hline & summer & $\mathrm{N}=17(20.2 \%)$ & $\mathrm{N}=5(17.2 \%)$ & $\mathrm{N}=12(21.8 \%)$ & \\
\hline & autumn & $\mathrm{N}=22(26.2 \%)$ & $\mathrm{N}=6(20.7 \%)$ & $\mathrm{N}=16(29.1 \%)$ & \\
\hline \multirow[t]{4}{*}{ Season - relapse } & winter & $\mathrm{N}=10(34.5 \%)$ & $\mathrm{N}=10(34.5 \%)$ & - & \\
\hline & spring & $\mathrm{N}=7(24.1 \%)$ & $\mathrm{N}=7(24.1 \%)$ & - & \\
\hline & summer & $\mathrm{N}=6(20.7 \%)$ & $\mathrm{N}=6(20.7 \%)$ & - & \\
\hline & autumn & $\mathrm{N}=6(20.7 \%)$ & $\mathrm{N}=6(20.7 \%)$ & - & \\
\hline Time to relapse - months & & $13.0(2.0 ; 128.9)$ & $13.0(2.0 ; 128.9)$ & - & \\
\hline Age & & $62.0(39.0 ; 74.0)$ & $62.0(29.0 ; 75.0)$ & $62.0(39.0 ; 74.0)$ & 0.598 \\
\hline \multirow[t]{2}{*}{ Age } & $\leq 65$ & $\mathrm{~N}=57(67.9 \%)$ & $\mathrm{N}=17(58.6 \%)$ & $\mathrm{N}=40(72.7 \%)$ & \multirow{2}{*}{0.192} \\
\hline & $>65$ & $\mathrm{~N}=27(32.1 \%)$ & $\mathrm{N}=12(41.4 \%)$ & $\mathrm{N}=15(27.3 \%)$ & \\
\hline \multirow[t]{2}{*}{ Gender } & $\mathrm{F}$ & $\mathrm{N}=35(41.7 \%)$ & $\mathrm{N}=13(44.8 \%)$ & $\mathrm{N}=22(40.0 \%)$ & \multirow{2}{*}{0.670} \\
\hline & $\mathrm{M}$ & $\mathrm{N}=49(58.3 \%)$ & $\mathrm{N}=16(55.2 \%)$ & $\mathrm{N}=33(60.0 \%)$ & \\
\hline BMI & & $26.0(19.4 ; 34.8)$ & $27.3(17.3 ; 35.8)$ & $25.7(20.2 ; 33.8)$ & 0.389 \\
\hline \multirow[t]{4}{*}{ BMI categories } & $\leq 20$ & $\mathrm{~N}=6(7.1 \%)$ & $\mathrm{N}=4(13.8 \%)$ & $\mathrm{N}=2(3.6 \%)$ & \multirow{4}{*}{0.105} \\
\hline & $21-25$ & $\mathrm{~N}=32(38.1 \%)$ & $\mathrm{N}=7(24.1 \%)$ & $\mathrm{N}=25(45.5 \%)$ & \\
\hline & $26-30$ & $\mathrm{~N}=24(28.6 \%)$ & $\mathrm{N}=8(27.6 \%)$ & $\mathrm{N}=16(29.1 \%)$ & \\
\hline & $>30$ & $\mathrm{~N}=22(26.2 \%)$ & $\mathrm{N}=10(34.5 \%)$ & $\mathrm{N}=12(21.8 \%)$ & \\
\hline \multirow[t]{5}{*}{ Stage } & 1 & $\mathrm{~N}=1(1.2 \%)$ & $\mathrm{N}=1(3.4 \%)$ & $\mathrm{N}=0(0.0 \%)$ & \multirow{5}{*}{-} \\
\hline & 2 & $\mathrm{~N}=6(7.1 \%)$ & $\mathrm{N}=6(20.7 \%)$ & $\mathrm{N}=0(0.0 \%)$ & \\
\hline & 3 & $\mathrm{~N}=21(25.0 \%)$ & $\mathrm{N}=21(72.4 \%)$ & $\mathrm{N}=0(0.0 \%)$ & \\
\hline & 4 & $\mathrm{~N}=55(65.5 \%)$ & $\mathrm{N}=0(0.0 \%)$ & $\mathrm{N}=55(100.0 \%)$ & \\
\hline & $\mathrm{x}$ & $\mathrm{N}=1(1.2 \%)$ & $\mathrm{N}=1(3.4 \%)$ & $\mathrm{N}=0(0.0 \%)$ & \\
\hline \multirow[t]{3}{*}{ KRAS } & mut & $\mathrm{N}=30(35.7 \%)$ & $\mathrm{N}=10(34.5 \%)$ & $\mathrm{N}=20(36.4 \%)$ & \multirow{3}{*}{0.947} \\
\hline & wt & $\mathrm{N}=41(48.8 \%)$ & $\mathrm{N}=14(48.3 \%)$ & $\mathrm{N}=27(49.1 \%)$ & \\
\hline & unknown & $\mathrm{N}=13(15.5 \%)$ & $\mathrm{N}=5(17.2 \%)$ & $\mathrm{N}=8(14.5 \%)$ & \\
\hline \multirow[t]{4}{*}{ Grade } & 1 & $\mathrm{~N}=11(15.9 \%)$ & $\mathrm{N}=7(29.2 \%)$ & $\mathrm{N}=4(8.9 \%)$ & \multirow{4}{*}{0.142} \\
\hline & 2 & $\mathrm{~N}=45(65.2 \%)$ & $\mathrm{N}=14(58.3 \%)$ & $\mathrm{N}=31(68.9 \%)$ & \\
\hline & 3 & $\mathrm{~N}=12(17.4 \%)$ & $\mathrm{N}=3(12.5 \%)$ & $\mathrm{N}=9(20.0 \%)$ & \\
\hline & 4 & $\mathrm{~N}=1(1.4 \%)$ & $\mathrm{N}=0(0.0 \%)$ & $\mathrm{N}=1(2.2 \%)$ & \\
\hline \multirow[t]{3}{*}{ Diagnosis } & C18.0-C18.4 & $\mathrm{N}=14(16.7 \%)$ & $\mathrm{N}=2(6.9 \%)$ & $\mathrm{N}=12(21.8 \%)$ & \multirow{3}{*}{0.108} \\
\hline & C18.5-C18.7 & $\mathrm{N}=21(25.0 \%)$ & $\mathrm{N}=10(34.5 \%)$ & $\mathrm{N}=11(20.0 \%)$ & \\
\hline & C18.8-C20 & $\mathrm{N}=49(58.3 \%)$ & $\mathrm{N}=17(58.6 \%)$ & $\mathrm{N}=32(58.2 \%)$ & \\
\hline \multirow[t]{4}{*}{ PS } & 0 & $\mathrm{~N}=15(17.9 \%)$ & $\mathrm{N}=5(17.2 \%)$ & $\mathrm{N}=10(18.2 \%)$ & \multirow{4}{*}{0.524} \\
\hline & 1 & $\mathrm{~N}=59(70.2 \%)$ & $\mathrm{N}=22(75.9 \%)$ & $\mathrm{N}=37(67.3 \%)$ & \\
\hline & 2 & $\mathrm{~N}=8(9.5 \%)$ & $\mathrm{N}=2(6.9 \%)$ & $\mathrm{N}=6(10.9 \%)$ & \\
\hline & 3 & $\mathrm{~N}=2(2.4 \%)$ & $\mathrm{N}=0(0.0 \%)$ & $\mathrm{N}=2(3.6 \%)$ & \\
\hline
\end{tabular}


Table 1. Basic description of patient cohort according to primary metastatic cancer (continued)

\begin{tabular}{|c|c|c|c|c|c|}
\hline \multirow{2}{*}{ Characteristics } & & \multirow[b]{2}{*}{ Total $(\mathrm{N}=84)$} & \multicolumn{2}{|c|}{ Primary metastatic disease } & \multirow{2}{*}{$\mathbf{p}^{1}$} \\
\hline & & & No $(\mathrm{N}=29)$ & Yes $(\mathrm{N}=55)$ & \\
\hline \multirow[t]{4}{*}{ MT - number } & 1 & $\mathrm{~N}=43(51.2 \%)$ & $\mathrm{N}=17(58.6 \%)$ & $\mathrm{N}=26(47.3 \%)$ & \multirow{4}{*}{0.792} \\
\hline & 2 & $\mathrm{~N}=25(29.8 \%)$ & $\mathrm{N}=7(24.1 \%)$ & $\mathrm{N}=18(32.7 \%)$ & \\
\hline & 3 & $\mathrm{~N}=13(15.5 \%)$ & $\mathrm{N}=4(13.8 \%)$ & $\mathrm{N}=9(16.4 \%)$ & \\
\hline & 4 & $\mathrm{~N}=3(3.6 \%)$ & $\mathrm{N}=1(3.4 \%)$ & $\mathrm{N}=2(3.6 \%)$ & \\
\hline \multirow[t]{2}{*}{ Loc. - liver } & no & $\mathrm{N}=25(29.8 \%)$ & $\mathrm{N}=18(62.1 \%)$ & $\mathrm{N}=7(12.7 \%)$ & \multirow{2}{*}{$<0.001$} \\
\hline & yes & $\mathrm{N}=59(70.2 \%)$ & $\mathrm{N}=11(37.9 \%)$ & $\mathrm{N}=48(87.3 \%)$ & \\
\hline \multirow[t]{2}{*}{ Loc. - peritoneum } & no & $\mathrm{N}=50(59.5 \%)$ & $\mathrm{N}=14(48.3 \%)$ & $\mathrm{N}=36(65.5 \%)$ & \multirow{2}{*}{0.129} \\
\hline & yes & $\mathrm{N}=34(40.5 \%)$ & $\mathrm{N}=15(51.7 \%)$ & $\mathrm{N}=19(34.5 \%)$ & \\
\hline \multirow[t]{2}{*}{ Loc. - lungs } & no & $\mathrm{N}=72(85.7 \%)$ & $\mathrm{N}=25(86.2 \%)$ & $\mathrm{N}=47(85.5 \%)$ & \multirow{2}{*}{0.925} \\
\hline & yes & $\mathrm{N}=12(14.3 \%)$ & $\mathrm{N}=4(13.8 \%)$ & $\mathrm{N}=8(14.5 \%)$ & \\
\hline \multirow[t]{2}{*}{ Loc. - other } & no & $\mathrm{N}=52(61.9 \%)$ & $\mathrm{N}=15(51.7 \%)$ & $\mathrm{N}=37(67.3 \%)$ & \multirow{2}{*}{0.165} \\
\hline & yes & $\mathrm{N}=32(38.1 \%)$ & $\mathrm{N}=14(48.3 \%)$ & $\mathrm{N}=18(32.7 \%)$ & \\
\hline \multirow[t]{2}{*}{ Any surgical procedure } & no & $\mathrm{N}=43(51.2 \%)$ & $\mathrm{N}=16(55.2 \%)$ & $\mathrm{N}=27(49.1 \%)$ & \multirow{2}{*}{0.596} \\
\hline & yes & $\mathrm{N}=41(48.8 \%)$ & $\mathrm{N}=13(44.8 \%)$ & $\mathrm{N}=28(50.9 \%)$ & \\
\hline \multirow[t]{2}{*}{ Radical resection } & no & $\mathrm{N}=68(81.0 \%)$ & $\mathrm{N}=22(75.9 \%)$ & $\mathrm{N}=46(83.6 \%)$ & \multirow{2}{*}{0.394} \\
\hline & yes & $\mathrm{N}=16(19.0 \%)$ & $\mathrm{N}=7(24.1 \%)$ & $\mathrm{N}=9(16.4 \%)$ & \\
\hline CT type & Length of $\mathrm{CT}^{2}$ & & & & \multirow{7}{*}{0.647} \\
\hline bev+FOLFOX & $10.0(9.3 ; 10.9)$ & $\mathrm{N}=7(8.3 \%)$ & $\mathrm{N}=2(6.9 \%)$ & $\mathrm{N}=5(9.1 \%)$ & \\
\hline bev+XELOX & $9.0(5.5 ; 16.3)$ & $\mathrm{N}=49(58.3 \%)$ & $\mathrm{N}=15(51.7 \%)$ & $\mathrm{N}=34(61.8 \%)$ & \\
\hline bFOL & $8.2(2.5 ; 9.1)$ & $\mathrm{N}=3(3.6 \%)$ & $\mathrm{N}=1(3.4 \%)$ & $\mathrm{N}=2(3.6 \%)$ & \\
\hline FOLFOX & $7.9(5.5 ; 10.7)$ & $\mathrm{N}=8(9.5 \%)$ & $\mathrm{N}=2(6.9 \%)$ & $\mathrm{N}=6(10.9 \%)$ & \\
\hline XELOX & $6.7(5.9 ; 10.4)$ & $\mathrm{N}=12(14.3 \%)$ & $\mathrm{N}=6(20.7 \%)$ & $\mathrm{N}=6(10.9 \%)$ & \\
\hline other & $5.9(3.1 ; 7.8)$ & $\mathrm{N}=5(6.0 \%)$ & $\mathrm{N}=3(10.3 \%)$ & $\mathrm{N}=2(3.6 \%)$ & \\
\hline Time to therapy cessation & & $8.5(3.1 ; 23.9)$ & $7.8(3.0 ; 16.3)$ & $8.8(3.1 ; 23.9)$ & 0.255 \\
\hline \multirow[t]{4}{*}{ Reason for therapy cessation } & remission & $\mathrm{N}=9(12.2 \%)$ & $\mathrm{N}=0(0.0 \%)$ & $\mathrm{N}=9(18.8 \%)$ & \multirow{4}{*}{0.001} \\
\hline & patient decision & $\mathrm{N}=8(10.8 \%)$ & $\mathrm{N}=5(19.2 \%)$ & $\mathrm{N}=3(6.3 \%)$ & \\
\hline & progression & $\mathrm{N}=46(62.2 \%)$ & $\mathrm{N}=13(50.0 \%)$ & $\mathrm{N}=33(68.8 \%)$ & \\
\hline & other & $\mathrm{N}=11(14.9 \%)$ & $\mathrm{N}=8(30.8 \%)$ & $\mathrm{N}=3(6.3 \%)$ & \\
\hline \multirow[t]{4}{*}{ Best response } & CR & $\mathrm{N}=16(19.0 \%)$ & $\mathrm{N}=3(10.3 \%)$ & $\mathrm{N}=13(23.6 \%)$ & \multirow{4}{*}{0.091} \\
\hline & $\mathrm{PD}$ & $\mathrm{N}=5(6.0 \%)$ & $\mathrm{N}=4(13.8 \%)$ & $\mathrm{N}=1(1.8 \%)$ & \\
\hline & PR & $\mathrm{N}=25(29.8 \%)$ & $\mathrm{N}=8(27.6 \%)$ & $\mathrm{N}=17(30.9 \%)$ & \\
\hline & SD & $\mathrm{N}=38(45.2 \%)$ & $\mathrm{N}=14(48.3 \%)$ & $\mathrm{N}=24(43.6 \%)$ & \\
\hline First clinically accessible levels ${ }^{3}$ : & 25-OHD & $30.4(17.7 ; 57.3)$ & $25.9(10.9 ; 57.3)$ & $31.5(18.6 ; 58.1)$ & 0.102 \\
\hline & $\mathrm{Ca}$ & $2.3(2.1 ; 2.6)$ & $2.3(2.1 ; 2.6)$ & $2.3(2.2 ; 2.6)$ & 0.399 \\
\hline & CEA & $6.8(0.9 ; 892.7)$ & $4.9(0.9 ; 701.6)$ & $9.2(0.6 ; 1242.0)$ & 0.556 \\
\hline & $\mathrm{Hb}$ & $126(98 ; 150)$ & $125(98 ; 150)$ & $130(97 ; 149)$ & 0.501 \\
\hline & Albumin & $41.0(35.0 ; 46.0)$ & $43.0(38.0 ; 49.0)$ & $41.0(34.0 ; 46.0)$ & 0.056 \\
\hline & eGFR & $1.5(0.9 ; 1.9)$ & $1.5(0.7 ; 2.0)$ & $1.6(1.0 ; 1.9)$ & 0.332 \\
\hline & Cholesterol total & $5.0(2.7 ; 7.7)$ & $4.7(2.3 ; 6.8)$ & $5.1(3.6 ; 8.6)$ & 0.086 \\
\hline & LDL cholesterol & $3.0(1.3 ; 4.6)$ & $2.8(0.8 ; 4.6)$ & $3.0(1.7 ; 5.6)$ & 0.393 \\
\hline & HDL cholesterol & $1.1(0.5 ; 1.7)$ & $1.1(0.4 ; 1.9)$ & $1.2(0.6 ; 1.7)$ & 0.724 \\
\hline & Triacylglycerols & $1.6(0.8 ; 3.9)$ & $1.6(0.6 ; 3.2)$ & $1.7(0.8 ; 4.2)$ & 0.335 \\
\hline & NORMAL: & $\mathrm{N}=40(47.6 \%)$ & $\mathrm{N}=14(48.3 \%)$ & $\mathrm{N}=26(47.3 \%)$ & \\
\hline 25-OHD time-course patterns & LOW-fluctuating & $\mathrm{N}=14(16.7 \%)$ & $\mathrm{N}=5(17.2 \%)$ & $\mathrm{N}=9(16.4 \%)$ & 0.984 \\
\hline & LOW-stable & $\mathrm{N}=30(35.7 \%)$ & $\mathrm{N}=10(34.5 \%)$ & $\mathrm{N}=20(36.4 \%)$ & \\
\hline
\end{tabular}

Legend: ${ }^{1}$ Mann-Whitney U test for continuous variables; maximum likelihood chi-square test for categorical variables; Units of "first clinically accessible" blood levels of analytes: plasma 25-OHD (25-hydroxyvitamin D) =nmol/l, serum CEA = ug/l, blood Hb = g/l, plasma albumin = g/l, plasma creatinine-based eGFR = ml/s, plasma calcium, cholesterols and triacylglycerols $=\mathrm{mmol} / \mathrm{l}$; note $-\mathrm{Li}$-heparin plasma and additive-free serum were used for biochemical analyses. ${ }^{2} \mathrm{The}$ chemotherapy regimen were as follows: FOLFOX (oxaliplatin $85 \mathrm{mg} / \mathrm{m}^{2} \mathrm{IV}$ day1; leucovorin $200 \mathrm{mg} / \mathrm{m}^{2} \mathrm{IV}$ days 1 and 2; 5-FU bolus $400 \mathrm{mg} / \mathrm{m}^{2} \mathrm{IV}$ days 1 and 2; 5-FU $600 \mathrm{mg} / \mathrm{m}^{2}$ IV 22-hour continuous infusion days 1 and 2 every 2 weeks) or XELOX (oxaliplatin $130 \mathrm{mg} / \mathrm{m}^{2} \mathrm{IV}$ day 1 ; capecitabine $1000 \mathrm{mg} / \mathrm{m}^{2}$ twice daily PO for 14 days every 3 weeks). bFOL (oxaliplatin $85 \mathrm{mg} / \mathrm{m}^{2} \mathrm{IV}$ days 1,15; leucovorin $20 \mathrm{mg} / \mathrm{m}^{2} \mathrm{IV}$ and 5-FU bolus $500 \mathrm{mg} / \mathrm{m}^{2} \mathrm{IV}$ days $1,8,15$ every 4 weeks. Bevacizumab was administered at a dosage of $5 \mathrm{mg} / \mathrm{kg}$ IV every 2 weeks or $7.5 \mathrm{mg} / \mathrm{kg}$ IV every 3 weeks depending on the chemotherapy regimen. ${ }^{3} \mathrm{Median}$ (inter-quartile range). 25 -OHD time-course patterns contains two types NORMAL and LOW, the LOW being presented here with its subtypes showing subtle but insignificant variations. 
information system besides blood levels of 25-OHD, calcium, albumin, total cholesterol, LDL-measured and HDL cholesterol that were additionally determined in tumour marker serum specimen biobanked aliquots [15] using standard clinical laboratory methods (25-OHD - Abbott Architect, all other - Roche Cobas Integra). Estimated glomerular filtration rates were calculated using CKDepi equations [16] based on Li-heparin plasma creatinine enzyme determination. The reference ranges of 25-OHD were derived in-house from a set of healthy individuals from South Moravian region. Complete blood cell count was determined using Sysmex XE5000 instrument. Patients were monitored using common clinical, laboratory and imaging methods and were not provided with any special procedure such as a clinical trial, special counselling services and/or dietary or vitamin supplements.

\section{Statistical methodology}

Standard descriptive statistics were applied in the analysis; absolute and relative frequencies for categorical variables and median estimates supplied with $5^{\text {th }}-95^{\text {th }}$ percentile range or mean and standard error for continuous variables. Statistical significance of differences between groups of patients was tested using maximum likelihood chi-square test for categorical variables and Mann-Whitney U test for continuous variables. Correlations between continuous variables were quantified and tested by means of Spearman rank correlation (robust non-parametric method) and Pearson correlation coefficients (parametric product-limit correlation). Progression-free survival and overall survival were taken as principal outcome endpoints of the study. Both the time-to-event endpoints were calculated since the onset of therapy of metastatic CRC. Timeto-event data sets were visualized using standard Kaplan-Meier methodology. Comprehensive set of statistics was derived from time series formed by biochemical parameters consecutively measured during therapy. We defined the "first clinically accessible levels" as those measured in the specimen for first CEA determination and denoted T01 in the subsequent time series as described in the Fig 1. Subsequent time series then provided estimates of min-max values, their ranges, relative median and maximum changes in time (related to the first clinically accessible level), integrated area under time-related concentration curve, and absolute changes per given time interval. All time-related statistics were subsequently examined as potential predictors of outcome endpoints. Quantitative biochemical indicators (both first accessible values and timeseries statistics) as potential predictors of survival endpoints were separately examined to define informative cut-off points. Effective cut-off values were optimized on the basis of Receiver Operating Characteristics curves [17]. The computation was based on binormal assumption. Standard measures of sensitivity and specificity were estimated and supported by $95 \%$ confidence intervals for each analyzed indicator and associated cut-off points [18]. The final set of potential prognostic factors and interaction terms coded as binary variables according to the cut-off points was subjected to Cox proportional hazards regression model and the prognostic power was quantified by hazard ratios and corresponding 95\% confidence intervals. Both univariate and multivariate regression models were applied, the latter using forward stepwise selection algorithm driven by maximum likelihood ratio test. An "alfa" value =

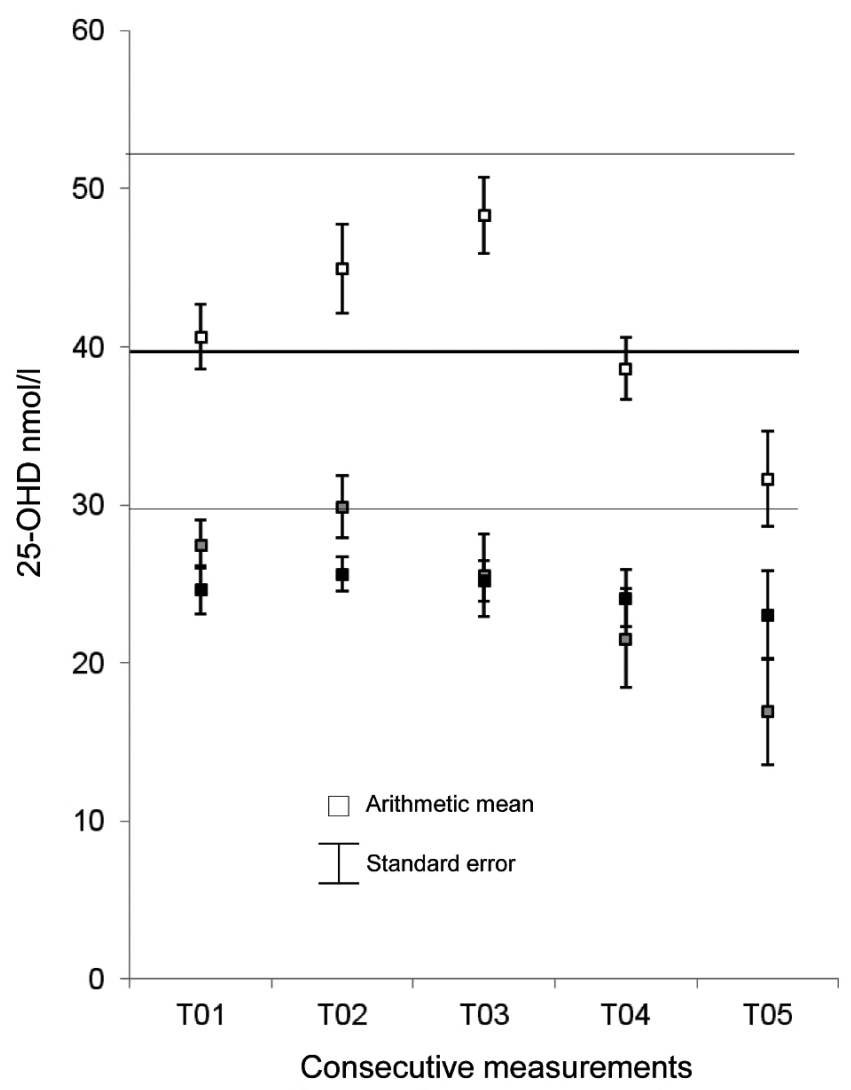

\begin{tabular}{lccccc} 
Number of samples in given time & T01 & T02 & T03 & T04 & T05 \\
\hline \\
\hline At least 1 25-OHD value 40 or above & 40 & 40 & 32 & 24 & 8 \\
\hline All 25-OHD < 40 - fluctuating & 14 & 14 & 12 & 9 & 6 \\
\hline All 25-OHD < 40 - stable & 30 & 30 & 15 & 11 & 7 \\
\hline
\end{tabular}

Time between biochemical sample and start of chemotherapy

\begin{tabular}{rrc}
\hline $\begin{array}{r}\text { Time between start of the therapy }{ }^{1} \text { and } \\
\text { "first clinically accessible value T01“ }\end{array}$ & $\mathbf{N}(\%)$ & $\begin{array}{c}\text { Cumulative } \\
\mathbf{N}(\%)\end{array}$ \\
\hline 0 months & $39(46.5 \%)$ & $39(46.4 \%)$ \\
$0-2$ month & $29(34.5 \%)$ & $68(80.9 \%)$ \\
$>2$ months & $16(19.0 \%)$ & $84(100.0 \%)$ \\
\hline
\end{tabular}

Figure 1. Time-course typology patterns of 25-OHD blood levels during therapy of metastatic CRC

Legend: ${ }^{1}$ absolute difference between date of biochemical sample and start of chemotherapy 
0.05 was used as limit of statistical significance in all performed analyses. Analyses were computed using SPSS 22 [19].

\section{Results}

Baseline overall characteristics of patients are presented in the Table 1 . The median age was 62 years (range $39-74$ ) with slight prevalence of males $(58.3 \%)$. The majority of patients were of performance status ECOG 0-1 (88.1\%). Synchronous primary and metastatic disease was seen in $65.4 \%$ of patients. We found no statistically significant differences in survival outcomes between primary metastatic and nonmetastatic disease (Fig. 2). Medians of progression free survival (PFS) and overall survival (OS) were 15.4 months for PFS and 41.2 months for OS, respectively. Predictors of PFS and OS not statistically significant are listed in the Legend to the Table 2. The cut-off levels of $40 \mathrm{nmol} / \mathrm{l}$ for 25-OHD and $11 \mathrm{ug} / \mathrm{l}$ for first CEA derived from the ROC analyses proved to be clinical decision levels for stratifying patients to the respective prognostic groups. Time-related blood levels of 25-OHD segregated in two typology patterns denoted in relation to the reference ranges as being NORMAL or LOW (Fig. 3) and along with "first CEA" and "patient surgery" consisting of either any surgical procedure or radical resection of metastases showed significant associations with disease outcomes (Table 2).

\section{Discussion}

In this study we confirmed fundamental vitamin $\mathrm{D}$ deficiency [20] in our set of metastatic colorectal cancer patients. As expected, our results showed the critical effect of surgery on OS and the strong predictive and prognostic power of CEA.

The overall host/disease responses to vitamin $\mathrm{D}$ and related compounds appear to be very complex involving host immunity modulation and VDR-dependent differentiating properties at the disease level as well. Brenner et al published a meta-analysis of prospective cohort studies in breast and CRC patients, concluding that 25-OHD levels higher than $75 \mathrm{nmol} / \mathrm{l}$ were associated with reduced mortality [21]. Very recently, $\mathrm{Ng}$ and coworkers presented data indicating possible associations of "postdiagnostic" blood levels of vitamin $\mathrm{D}$ with disease outcomes in a set of North American patients [22]. The published reports analyzed mostly single-sampled "prediagnostic or postdiagnostic" levels of 25-OHD and were
Progression free survival

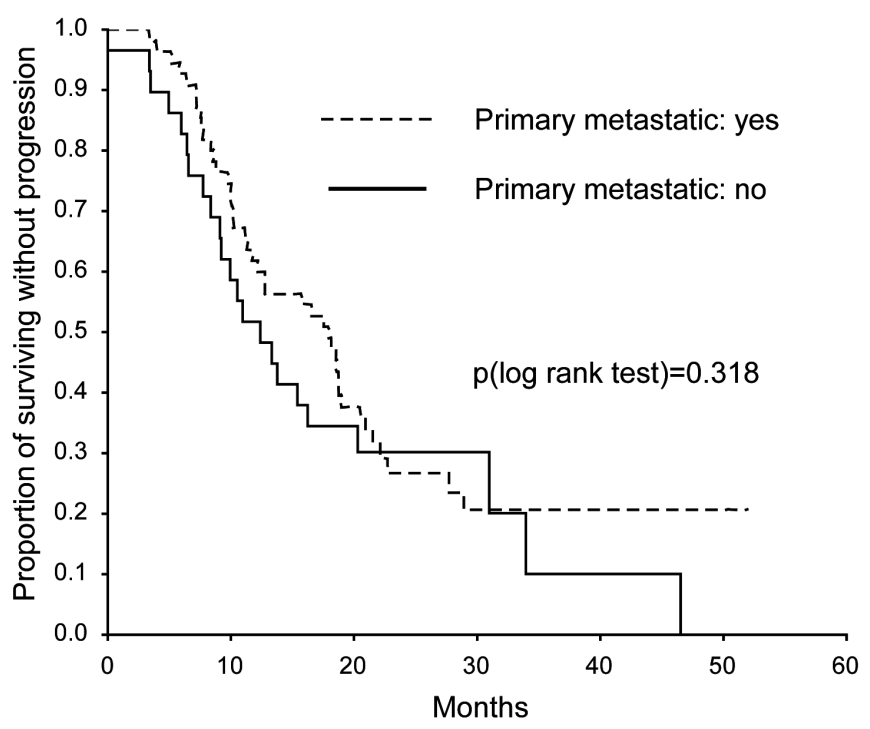

\begin{tabular}{ccccc}
\hline & \multicolumn{2}{c}{ Primary metastatic: no } & \multicolumn{2}{c}{ Primary metastatic: yes } \\
Time & $\begin{array}{c}\text { No at } \\
\text { risk }\end{array}$ & Surviving $(\mathbf{9 5 \%}$ Cl) & $\begin{array}{c}\text { No at } \\
\text { risk }\end{array}$ & Surviving (95\% Cl) \\
\hline 0 & 29 & 1.000 & 55 & 1.000 \\
12 & 15 & $0.517(0.335 ; 0.699)$ & 34 & $0.618(0.490 ; 0.747)$ \\
24 & 5 & $0.302(0.131 ; 0.472)$ & 11 & $0.267(0.144 ; 0.389)$ \\
36 & 1 & $0.101(0.007 ; 0.271)$ & 2 & $0.207(0.087 ; 0.327)$ \\
48 & 0 & 0.000 & 2 & $0.207(0.087 ; 0.327)$ \\
\hline
\end{tabular}

\section{Overall survival}

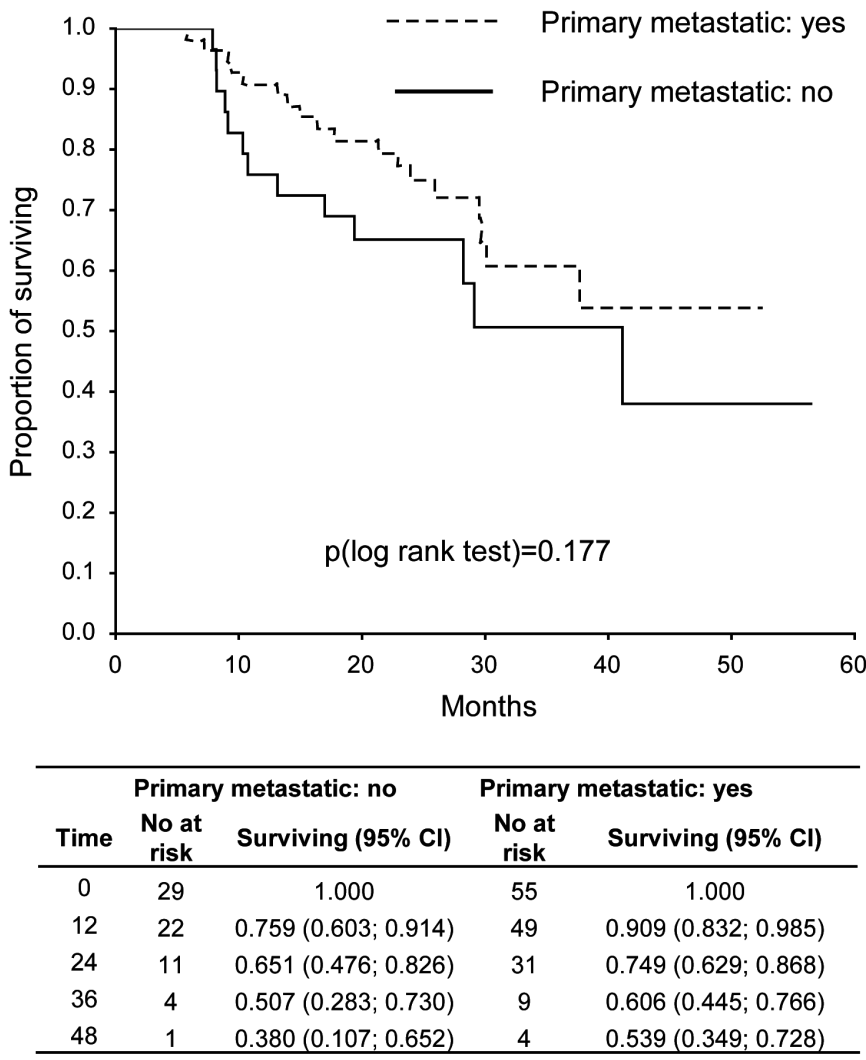

Figure 2. Survival of patients stratified according to primary diagnosis of CRC

Legend: No statistically significant difference in survival outcomes between primary metastatic and nonmetastatic disease was observed. 
--- NORMAL (N)

LOW (L)
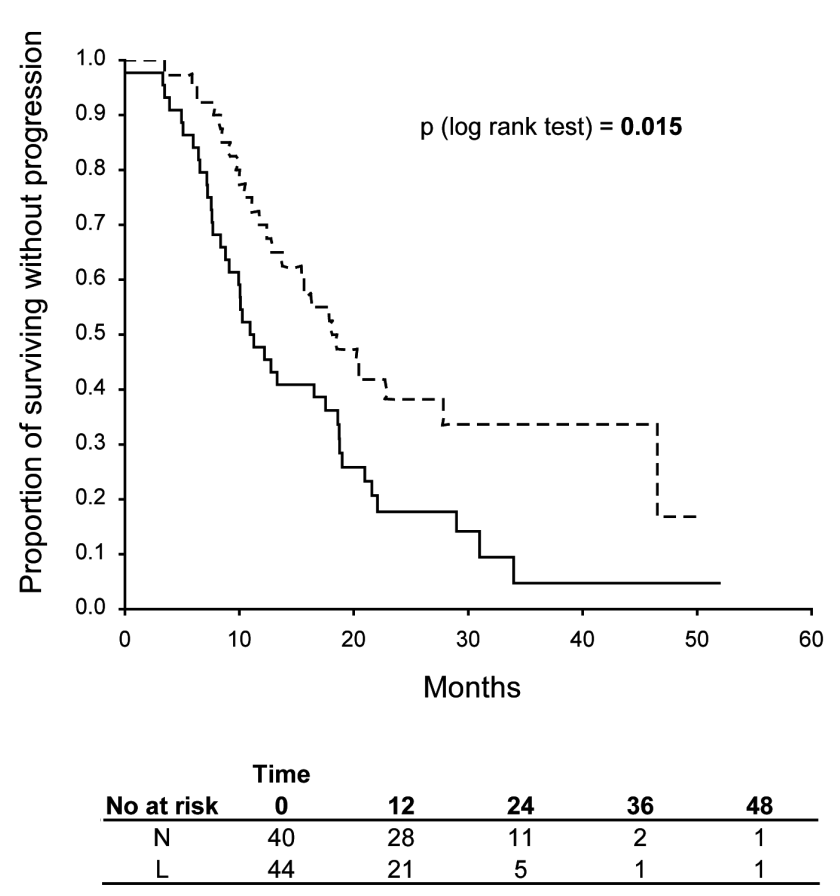

(N): At least one $25-\mathrm{OHD}$ value 40 or above $(\mathrm{N}=40)$

(L): All 25-OHD < $40(\mathrm{~N}=44)$

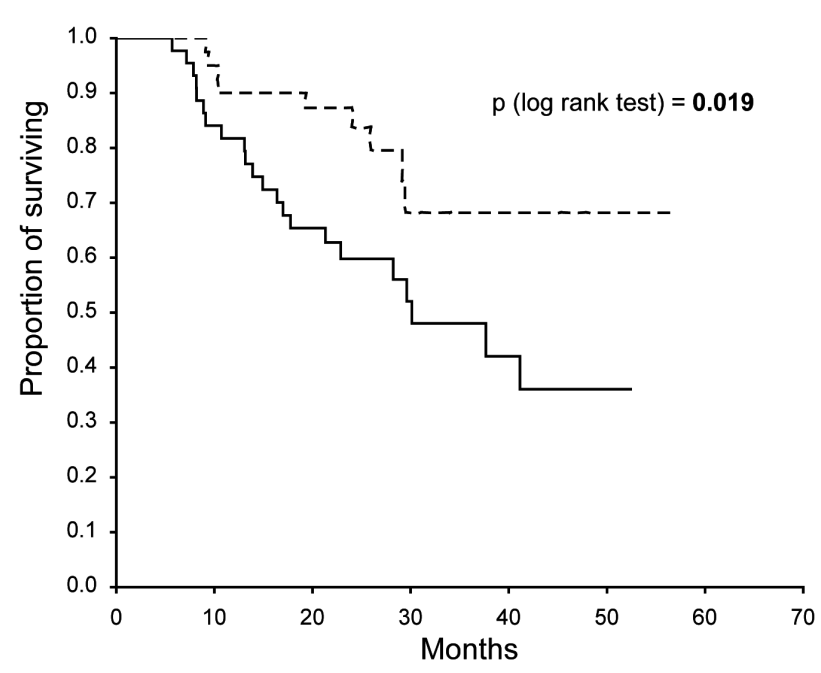

Figure 3. Survival of patients according to time-course typology patterns of 25-OHD blood levels (nmol/l)

Legend: Blood levels of 25-OHD showing typology patterns classified against the local winter (i.e. lower) reference ranges as being NORMAL or LOW; the LOW group showed subtle variations as "All $25-\mathrm{OHD}<40$ - fluctuating" (meaning any blood level elevation relative to the previous sampling i.e. due to improved conditions because of response to treatment), and "All $25-\mathrm{OHD}<40$ - stable" (meaning profound, consistently low levels)

Table 2. Potential predictors of PFS and OS in Cox proportional hazard regression model

\begin{tabular}{|c|c|c|c|c|c|}
\hline \multirow{2}{*}{ Endpoint: progression free survival } & & \multicolumn{2}{|c|}{ Univariate estimates } & \multicolumn{2}{|c|}{ Multivariate-adjusted estimates } \\
\hline & & $\mathrm{HR}(95 \% \mathrm{CI})$ & $\mathbf{p}$ & HR $(95 \% \mathrm{CI})$ & $\mathbf{p}$ \\
\hline Grade & $\geq 2$ & $2.449(1.077 ; 5.567)$ & 0.033 & & \\
\hline $\mathrm{N}$ & $\mathrm{N} 1+\mathrm{N} 2$ & $2.159(1.081 ; 4.698)$ & 0.045 & & \\
\hline \multirow[t]{2}{*}{ 25-hydroxyvitamin D (25-OHD), nmol/l } & $25-\mathrm{OHD}(\max )<40$ & $1.881(1.131 ; 3.129)$ & 0.014 & & \\
\hline & $25-\mathrm{OHD}(\max )<50$ & $2.055(1.131 ; 3.739)$ & 0.018 & & \\
\hline All 25-OHD in time series $<50$ & & $2.013(1.124 ; 3.605)$ & 0.019 & & \\
\hline All $25-\mathrm{OHD}$ in time series $<40$ & & $1.841(1.116 ; 3.038)$ & 0.017 & & \\
\hline Typology of 25-OHD time series (binary code) & All values $<40(\mathrm{LOW})$ & $1.637(1.053 ; 2.544)$ & 0.031 & $1.699(1.016 ; 2.845)$ & 0.043 \\
\hline \multirow[t]{2}{*}{ CEA ug/l } & CEA (first) $>11$ & $2.226(1.355 ; 3.657)$ & 0.002 & $2.060(1.247 ; 3.402)$ & 0.004 \\
\hline & $\mathrm{CEA}(\max )>11$ & $2.345(1.141 ; 3.897)$ & 0.001 & & \\
\hline \multicolumn{6}{|l|}{ Endpoint: overall survival } \\
\hline Any surgical procedure & & $0.239(0.098 ; 0.583)$ & 0.002 & $0.240(0.097 ; 0.594)$ & 0.002 \\
\hline Radical resection of metastases & & $0.101(0.014 ; 0.747)$ & 0.025 & & \\
\hline 25-hydroxy D, in nmol/l & $25-\mathrm{OHD}(\max )<40$ & $2.238(1.029 ; 4.868)$ & 0.042 & & \\
\hline $25-\mathrm{OHD}(\max )<50$ & & $3.178(1.107 ; 9.068)$ & 0.032 & & \\
\hline All 25-OHD in time series $<50$ & & $3.344(1.168 ; 9.576)$ & 0.024 & & \\
\hline All 25-OHD in time series $<40$ & & $2.466(1.133 ; 5.371)$ & 0.023 & & \\
\hline Typology of 25-OHD time series (binary code) & All values < $40 \mathrm{LOW}$, & $2.710(1.333 ; 5.510)$ & 0.006 & $2.220(1.074 ; 4.592)$ & 0.031 \\
\hline CEA ug/l & First CEA > 11 & $4.610(2.115 ; 10.051)$ & $<0.001$ & $4.862(2.196 ; 10.762)$ & $<0.001$ \\
\hline
\end{tabular}

Table 2 Potential predictors of PFS and OS in Cox proportional hazard regression model

Predictors of PFS not statistically significant: season - diagnosis and relapse, age over 65 yrs, male gender, BMI, stage 3+4, KRAS, T, M1, diagnosis, type of disease (disseminated relapse or primary metastatic), PS, MT - number, localisation (liver, peritoneum, lungs, other), any surgical procedure, radical metastases resection, chemotherapy type, levels of calcium, haemoglobin, albumin, eGFR, cholesterol total, LDL cholesterol, HDL cholesterol, triacylglycerols.

Predictors of OS not statistically significant: season - diagnosis and relapse, age over 65 yrs, male gender, BMI, stage 3+4, KRAS, grade, T, N, M1, diagnosis, type of disease (disseminated relapse or primary metastatic), MT - number, localisation (liver, peritoneum, lungs, other), chemotherapy type, levels of calcium, haemoglobin, albumin, eGFR, cholesterol total, LDL cholesterol, HDL cholesterol, triacylglycerols. 
unable to show consistent associations with PFS and OS [14, $23,24]$. In fact, their results may be well in accordance with our "first clinically accessible levels" that did not show either enough predictive or prognostic power in our statistical models - by definition, our "first clinically accessible value" is either "prediagnostic or early postdiagnostic". Our reason for this design was that repetitive sampling may neutralize seasonal variation, better reflect the patient's premorbid period and interim clinical status and provide time course of blood concentration data. Furthermore, such a design is an essential component for the planned interventional clinical trial on supplementing vitamin $\mathrm{D}$ to personalize vitamin $\mathrm{D}$ dosing to the desired target levels.

To make the analysis feasible in this limited size cohort, we had to set forth some initial assumptions. Considering daily clinical practice, one finds it rather unfeasible to obtain consistent "true initial" concentrations. To overcome this, we instead defined "the 25-OHD first clinically accessible value" linked to the first determination of CEA constituting the $\mathrm{T}_{01}$ observation that became then consistent among the patients with acceptable variability (Fig. 1). The most informative outcome predictors were patient surgery, CEA and, independently, time-related blood levels of 25-OHD demonstrating that all patients with at least one level above $40 \mathrm{nmol} / \mathrm{l}$ and all below behaved differently when associated towards disease outcome. Neither the primary stage of the disease nor the time to metastatic stage interacted with variations and final predictive power of 25-OHD, implying that the time-course pattern of 25-OHD is a true independent prognostic factor of survival outcome. Nevertheless, our data should be interpreted with caution because of the limited cohort size even though our patient set was internally consistent, reflected clinical daily practice well and was free from apparent selection bias.

Our results imply that cholecalciferol may be proposed as an adjuvant component of treatment protocols in colorectal cancer irrespective of mechanisms leading to its deficiency. We suggest that the phenomenon of vitamin D deficiency may carry a therapeutic potential that, when possibly corrected, may enhance vitamin D-dependent biomodulation of host anticancer response.

Acknowledgements: supported by: Ministry of Education of the Czech Republic grants: LM2010004, LO1413 and LM2011017; Ministry of Health of the Czech Republic institutional support MH DRO 00209805

\section{References}

[1] NG K, WOLPIN BM, MEYERHARDT JA, WU K, CHAN AT, et al. (2009) Prospective study of predictors of vitamin D status and survival in patients with colorectal cancer. Br J Cancer 101: 916-923. http://dx.doi.org/10.1038/sj.bjc.6605262

[2] GARLAND CF, GARLAND FC (2006) Do sunlight and vitamin D reduce the likelihood of colon cancer? Int J Epidemiol 35: 217-220. http://dx.doi.org/10.1093/ije/dyi229
[3] GIOVANNUCCI E, LIU Y, RIMM EB, HOLLIS BW, FUCHS CS, et al. (2006) Prospective study of predictors of vitamin D status and cancer incidence and mortality in men. J Natl Cancer Inst 98: 451-459. http://dx.doi.org/10.1093/inci/dij101

[4] GORHAM ED, GARLAND CF, GARLAND FC, GRANT WB, MOHR SB, et al. (2007) Optimal vitamin D status for colorectal cancer prevention: a quantitative meta analysis. Am J Prev Med 32: 210-216. http://dx.doi.org/10.1016/j. amepre.2006.11.004

[5] APPERLY FL (1941) The relation of solar radiation to cancer mortality in North America. Cancer Res 1: 191-195.

[6] BEER TM (2003) Development of weekly high-dose calcitriol based therapy for prostate cancer. Urol Oncol 21: 399-405. http://dx.doi.org/10.1016/S1078-1439(03)00170-4

[7] FESKANICH D, MA J, FUCHS CS, KIRKNER GJ, HANKINSON SE, et al. (2004) Plasma vitamin D metabolites and risk of colorectal cancer in women. Cancer Epidemiol Biomarkers Prev 13: 1502-1508.

[8] STERBA J, VALIK D, MUDRY P, KEPAK T, PAVELKA Z, et al. (2006) Combined biodifferentiating and antiangiogenic oral metronomic therapy is feasible and effective in relapsed solid tumors in children: single-center pilot study. Onkologie 29: 308-313. http://dx.doi.org/10.1159/000093474

[9] CREW KD, GAMMON MD, STECK SE, HERSHMAN DL, CREMERS S, et al. (2009) Association between plasma 25hydroxyvitamin D and breast cancer risk. Cancer Prev Res (Phila) 2: 598-604. http://dx.doi.org/10.1158/1940-6207. CAPR-08-0138

[10] DEEB KK, TRUMP DL, JOHNSON CS (2007) Vitamin D signalling pathways in cancer: potential for anticancer therapeutics. Nat Rev Cancer 7: 684-700. http://dx.doi.org/10.1038/nrc2196

[11] HOLICK MF (2007) Vitamin D deficiency. N Engl J Med 357: 266-281. http://dx.doi.org/10.1056/NEJMra070553

[12] ROBSAHM TE, TRETLI S, DAHLBACK A, MOAN J (2004) Vitamin D3 from sunlight may improve the prognosis of breast-, colon- and prostate cancer (Norway). Cancer Causes Control 15: 149-158. http://dx.doi.org/10.1023/ B:CACO.0000019494.34403.09

[13] MOAN J, POROJNICU AC, ROBSAHM TE, DAHLBACK A, JUZENIENE A, et al. (2005) Solar radiation, vitamin $\mathrm{D}$ and survival rate of colon cancer in Norway. J Photochem Photobiol B 78: 189-193. http://dx.doi.org/10.1016/j. jphotobiol.2004.11.004

[14] NG K, SARGENT DJ, GOLDBERG RM, MEYERHARDT JA, GREEN EM, et al. (2011) Vitamin D status in patients with stage IV colorectal cancer: findings from Intergroup trial N9741. J Clin Oncol 29: 1599-1606. http://dx.doi.org/10.1200/ LCO.2010.31.7255

[15] HOLUB P, GREPLOVA K, KNOFLICKOVA D, NENUTIL R, VALIK D (2012) The biobanking research infrastructure BBMRI_CZ: a critical tool to enhance translational cancer research. Klin Onkol 25 Suppl 2: 2S78-81.

[16] LEVEY AS, STEVENS LA, SCHMID CH, ZHANG YL, CASTRO AF, 3RD, et al. (2009) A new equation to estimate glomerular filtration rate. Ann Intern Med 150: 604-612. http://dx.doi.org/10.7326/0003-4819-150-9-200905050$\underline{00006}$ 
[17] ENG J ROC analysis: web-based calculator for ROC curves. Baltimore: Johns Hopkins University.

[18] NEWCOMBE RG (1998) Two-sided confidence intervals for the single proportion: comparison of seven methods. Stat Med 17: 857-872. http://dx.doi.org/10.1002/(SICI)1097-0258(19980430)17:8<857::AID-SIM777>3.0.CO;2-E

[19] IBM_CORP (2013) IBM SPSS Statistics for Windows. Version 22.0. Armonk, NY: IBM Corp.

[20] NICOL AJ, TOKUYAMA H, MATTAROLLO SR, HAGI T, SUZUKI K, et al. (2011) Clinical evaluation of autologous gamma delta $\mathrm{T}$ cell-based immunotherapy for metastatic solid tumours. Br J Cancer 105: 778-786. http://dx.doi.org/10.1038/ bjc. 2011.293

[21] MAALMI H, ORDONEZ-MENA JM, SCHOTTKER B, BRENNER H (2014) Serum 25-hydroxyvitamin D levels and survival in colorectal and breast cancer patients: system- atic review and meta-analysis of prospective cohort studies. Eur J Cancer 50: 1510-1521. http://dx.doi.org/10.1016/j. ejca.2014.02.006

[22] NG K, VENOOK AP, SATO K, HOLLIS BW, NIEDZWIECKI D, et al. (2015) Vitamin D status and survival of metastatic colorectal cancer patients: Results from CALGB/SWOG 80405 (Alliance). J Clin Oncol 33.

[23] FEDIRKO V, RIBOLI E, TJONNELAND A, FERRARI P, OLSEN A, et al. (2012) Prediagnostic 25-hydroxyvitamin $\mathrm{D}, \mathrm{VDR}$ and CASR polymorphisms, and survival in patients with colorectal cancer in western European ppulations. Cancer Epidemiol Biomarkers Prev 21: 582-593. http://dx.doi. org/10.1158/1055-9965.EPI-11-1065

[24] NG K, MEYERHARDT JA, WU K, FESKANICH D, HOLLIS BW, et al. (2008) Circulating 25-hydroxyvitamin $\mathrm{d}$ levels and survival in patients with colorectal cancer. J Clin Oncol 26: 2984-2991. http://dx.doi.org/10.1200/ JCO.2007.15.1027 